Service social

\title{
Communication dans la relation d'aide, par Gérard Egan, Montréal, Éditions H.R.W., 1987. Manuel, 420 pages et cahier d'exercices, 140 pages. Traduction de Françoise Forest.
}

\section{Germain Trottier}

Volume 36, numéro 1, 1987

L’informatique dans les services sociaux

URI : https://id.erudit.org/iderudit/706356ar

DOI : https://doi.org/10.7202/706356ar

Aller au sommaire du numéro

Éditeur(s)

École de service social de l'Université Laval

ISSN

1708-1734 (numérique)

Découvrir la revue

Citer ce compte rendu

Trottier, G. (1987). Compte rendu de [Communication dans la relation d'aide, par Gérard Egan, Montréal, Éditions H.R.W., 1987. Manuel, 420 pages et cahier d'exercices, 140 pages. Traduction de Françoise Forest.] Service social, 36(1), 183-184. https://doi.org/10.7202/706356ar d'utilisation que vous pouvez consulter en ligne.

https://apropos.erudit.org/fr/usagers/politique-dutilisation/ 
L'avant-dernier chapitre présente, à l'aide de témoignages de médecins, d'infirmières et de travailleurs sociaux, le rôle des intervenants auprès des parents. Le dernier chapitre décrit les programmes et les services actuellement disponibles pour venir en aide aux enfants et à leur famille. Cette partie est suivie d'une liste des centres d'accueil de réadaptation et des centres de services sociaux des régions du Québec.

Le message livré par l'auteure aux intervenants est le suivant : apprivoisez vos propres sentiments face à la douleur, réfléchissez à vos propres attitudes et comportements vis-à-vis les personnes en situation de crise et « apprenez à vivre, à écouter et à respecter les autres, à vous informer et à informer les autres, à accompagner et à orienter plutôt qu'à diriger et contrôler ».

Ce volume présente les difficultés reliées à la naissance d'un enfant handicapé ainsi que les interventions, les programmes et les services offerts aux parents et aux jeunes enfants. II n'insiste pas sur les préjugés sociaux qu'ils auront à affronter, sur les barrières qui subsistent malgré les changements accomplis ces dernières années et sur les choix difficiles qui se poseront à l'âge adulte. Les témoignages présentés démontrent toutefois que les efforts quotidiens des parents sont profitables, tant pour l'enfant que pour eux-mêmes : ils découvrent en eux et dans leur entourage des ressources insoupçonnées pour affronter les difficultés au fur et à mesure qu'elles se présentent.

\section{Gaby CARRIER}

Centre de recherche sur les services communautaires, Université Laval.

Communication dans la relation d'aide, par Gérard Egan, Montréal, Éditions H.R.W., 1987. Manuel, 420 pages et cahier d'exercices, 140 pages. Traduction de Françoise Forest.

Il s'agit de la traduction de The Skilled Helper - A Systematic Approach Effective Helping, Belmont (Cal.), Brooks/Cole, 1986, troisième édition.

L'ouvrage vise à améliorer l'efficacité de la relation aidant/aidé. On le présente comme un modèle de pratique en contexte d'aide privilégiant la perspective humaniste. Il est composé de douze chapitres subdivisés en parties sensiblement égales, traitant tour à tour des aspects suivants: la remise en question de la relation d'aide, l'esquisse d'un modèle d'aide, les habiletés fondamentales en communication et le processus d'aide étape par étape. Il est complété par un cahier d'exercices de cent quarante pages qui sert d'outil d'accompagnement pour l'apprentissage des habiletés qui y sont retenues.

Cette traduction propose un modèle d'aide qui comporte un certain nombre de caractéristiques intéressantes que nous tenons à souligner.

Le modèle est axé sur une démarche de résolution de problème, ce qui le rend accessible à divers types de professionnels s'inspirant de cette approche dans leur pratique professionnelle. La valeur de la démarche d'aide centrée sur 
la résolution de problèmes a été éprouvée et tend à se généraliser. L'auteur précise, à bon droit, que "le monde entier peut être un laboratoire propice à la résolution de problèmes". À cet égard, l'axe retenu nous paraît un choix judicieux.

Le modèle proposé tente d'intégrer diverses théories et techniques en vue de favoriser une vision interdisciplinaire de l'aide. À notre avis, l'auteur n'y parvient pas tant qu'il le prétend mais, au moins, devons-nous lui reconnaître le mérite d'avoir tenté de le faire de façon pertinente et raisonnable. La recherche d'une approche intégrée intéresse et préoccupe les intervenants en relation d'aide, mais la façon de le faire reste encore à découvrir, à notre avis. L'ouvrage ici recensé doit être considéré, selon nous, comme une bonne contribution bien qu'incomplète et insatisfaisante en ce qui concerne, du moins, son application à la discipline du service social.

Le processus d'aide étape par étape est développé en rapport à la démarche de base. Les fondements sont bien établis et les éléments constitutifs s'y retrouvent présents. Toutefois, nous notons que cette démarche demeure à son niveau le plus élémentaire et ne comporte pas de complexités détaillées qui font souvent l'objet réel des impasses professionnelles dans la relation d'aide. En ce sens, ce volume donne accès à une compréhension générale du processus d'aide mais il ne précise pas comment dénouer les intrigues thérapeutiques courantes entre l'aidant et l'aidé dans la vie professionnelle de tous les jours.

Un fort accent est mis sur l'application logique de la démarche séquentielle. En pratique, les intervenants de la relation d'aide savent bien que la dynamique thérapeutique ne suit pas nécessairement l'ordre théorique mentionné, ce que souligne aussi l'auteur. L'application concrète et immédiate du processus proposé rend l'ouvrage particulièrement vivant et d'actualité. L'aidant peut l'utiliser comme une source de référence rapide et directe.

Le cahier d'exercices est un instrument complémentaire fort valable. Bien conçu, bien adapté, d'une présentation claire et nette, il permet à l'aidant en apprentissage de commencer une expérimentation de l'exercice professionnel de la communication d'aide. À noter toutefois qu'il s'agit d'un apprentissage d'habiletés fondamentales qui a besoin d'être renforcé par des outils supplémentaires de même nature. Par ailleurs, le contenu sur certaines attitudes a besoin d'être ajusté à la hausse pour donner leur portée véritable.

La bibliographie est abondante et constitue une banque de références utiles et faciles à repérer. Il en va de même pour l'index des auteurs et des sujets traités.

En somme, il s'agit d'un ouvrage de base bien traduit, bien conçu et recommandable pour l'apprentissage général à la communication dans la relation d'aide. L'utilité du modèle proposé pour l'enseignement universitaire du service social nous apparaît importante mais toutefois insuffisante en raison de la spécificité du domaine et de la complexité même des problèmes sociaux.

École de service social,

Germain Trottier Université Laval. 